\title{
Severe Hypotonia, Nystagmus and Hypomyelination in A 9-Month Female Infant: Diagnosing Pelizaeus - Merzbacher Disease Outside the Usual Inheritance Patterns
}

\author{
Panagiotis Krepis ${ }^{1 *}$, Maria Nikolaidou ${ }^{2}$, Despina Maritsi ${ }^{1}$, Julien Masliah-Planchon ${ }^{3}$, Odile Boespflug-Tanguy ${ }^{4}$, Celine Dupont ${ }^{5}$ and George Vartzelis ${ }^{1}$ \\ ${ }^{1}$ Second Department of Pediatrics, P\&A Kyriakou Children's Hospital, National and Kapodistrian University of Athens, Athens, Greece \\ ${ }^{2}$ Department of Neonatology, Elena Venizelou General Maternity Hospital, Athens, Greece \\ ${ }^{3}$ Oncogenetic Department, Institute of Curie, Paris, France \\ ${ }^{4}$ Departement of Neuropediatrics and Metabolic Diseases, Robert Debré Hospital, Paris, France \\ ${ }^{5}$ Department of Cytogenetics, Hospital Robert Debré, AP-HP, Paris, France
}

"Corresponding author: Panagiotis Krepis, Department of Pediatrics, P\&A Kyriakou Children's Hospital, National and Kapodistrian University of Athens, Athens, Greece, Tel: +30 210727 7000; E-mail: panoskr27@gmail.com

Rec date: Sep 05, 2016; Acc date: Sep 19, 2016; Pub date: Sep 25, 2016

Copyright: @ 2016 Krepis $\mathrm{P}$, et al. This is an open-access article distributed under the terms of the Creative Commons Attribution License, which permits unrestricted use, distribution, and reproduction in any medium, provided the original author and source are credited.

\begin{abstract}
Pelizaeus-Merzbacher disease is a rare X-linked recessive disorder regarding the defective myelin sheath formation in the CNS neurons due to mutations of the proteolipid protein 1 gene (PLP1). Even though it is predominant in males, affected females have been found to represent a small proportion in the medical literature 1. A wide variety of PLP1 mutations have been reported as a cause. Herein, we describe the case of a 9-month-old female with (bearing) the typical features of the disease who was found to have a rare mutation.
\end{abstract}

Keywords: Pelizaeus-Merzbacher; Female; Hypotonia; Nystagmus; Hypomyelination

\section{Introduction}

Pelizaeus-Merzbacher disease (PMD) is an inherited hypomyelination disorder which affects primarily the central nervous system. It is caused by genomic alterations of the proteolipid protein 1 gene $(P L P 1)$ resulting in aberrant function of oligodendrocytes. PMD is inherited as an $\mathrm{X}$-linked recessive trait. Males with PLP1 mutations manifest the disease, while females are usually unaffected carriers [1-3].

\section{Case Report}

We present the case of a 9-month-old female infant, the third child of her nonconsanguineous and healthy parents. She presented with severe head and truncal hypotonia impeding her feeding, as well as horizontal nystagmus which was noticed during the second month of life. Her parents reported a significant delay in the motor developmental milestones. She had achieved rolling at 8 months of age and she was unable to hold her head up, or sit, even with support. Nevertheless, she had good social interaction as she had developed social smile, she was recognizing familiar faces and she was babbling. Her past medical history-including pregnancy and perinatal period and family history were unremarkable. On physical examination, she was awake, smiling and making good eye contact. Spontaneous nystagmus was present bilaterally in horizontal direction. She also had severe axial hypotonia resulting in flaccidity. No hyperreflexia or clonus were present at that moment. No other abnormalities including dysmorphic features were noticed. In view of the clinical findings a magnetic resonance imaging (MRI) of the brain was carried out and revealed a severe delay in the myelination process, equivalent to 4 months of age (Figure 1A).

An extended genetic testing was performed at that moment, considering the classical PMD clinical features of nystagmus, hypotonia and ataxia along with the MRI findings. Copy number analysis of the PLP1 locus using multiplex ligation-dependent probe amplification (MLPA) revealed duplication of the entire gene. In order to explain the complete clinical expression of an X-linked recessive disease in a female patient, $\mathrm{X}$ chromosome inactivation studies were performed via methylation-sensitive restriction digest followed by PCR and fragment analysis. Unfavorable skewing of $\mathrm{X}$ inactivation, where the $\mathrm{X}$ chromosome carrying the mutant PLP1 allele is the predominantly active $\mathrm{X}$ in the patient was inferred to have a major role. Nonetheless, studies did not reveal a significant skewed pattern for any of the four X chromosome loci explored (AR, PCSK1N, SLITRK4, ZDHHC15). Therefore, further investigation was required in order to determine the mechanism responsible for the girl's phenotype.

Array CGH (aCGH) was performed, using the Agilent software eArray (GRCh37/hg19 assembly) with 30,032 probes, and confirmed a $752 \mathrm{kB}$ duplicated genomic region of chromosome Xq22, including the PLP1 gene. In addition, FISH analysis showed that this duplicated region was inserted in chromosome $1 \mathrm{p} 36$ (Figure 2A). The latter was finally affirmed by genome walking molecular method which targeted the specific position of Xq22 incorporation adjacent to the $1 \mathrm{p} 36$ chromosome of the patient (Figure 2B).

The condition of the girl improved slowly though she continued to have significant hypotonia and delayed motor development (holding her head up at 12 months, sitting with support at 16 months, standing at 30 months with knee immobilizer and cruising at 36 months). Her psychosocial interactions were excellent and her nystagmus improved. A repeat brain MRI at 33 months of age confirmed the diffuse 
Citation: Krepis P, Nikolaidou M, Maritsi D, Masliah-Planchon J, Boespflug-Tanguy O, et al. (2016) Severe Hypotonia, Nystagmus and Hypomyelination in A 9-Month Female Infant: Diagnosing Pelizaeus - Merzbacher Disease Outside the Usual Inheritance Patterns. J Neurol Disord 4: 301. doi:10.4172/2329-6895.1000301

Page 2 of 3

hypomyelinating pattern of the supratentorial cerebral white matter (Figure 1B). Furthermore, brainstem evoked potentials indicated a CNS conduction deficit. Physiotherapy and occupational therapy since the beginning of symptoms enhanced her motor skills so that she was able to crawl and walk with support at the age of [4]. In addition, her cognitive and language capacities were further promoted as she could speak with simple and comprehensive sentences. Nonetheless, progressively, signs of pyramidal tract dysfunction (spasticity, clonus, brisk tendon reflexes) became apparent and more discernible in the lower limbs in keeping with the typical symptomatology of PMD.

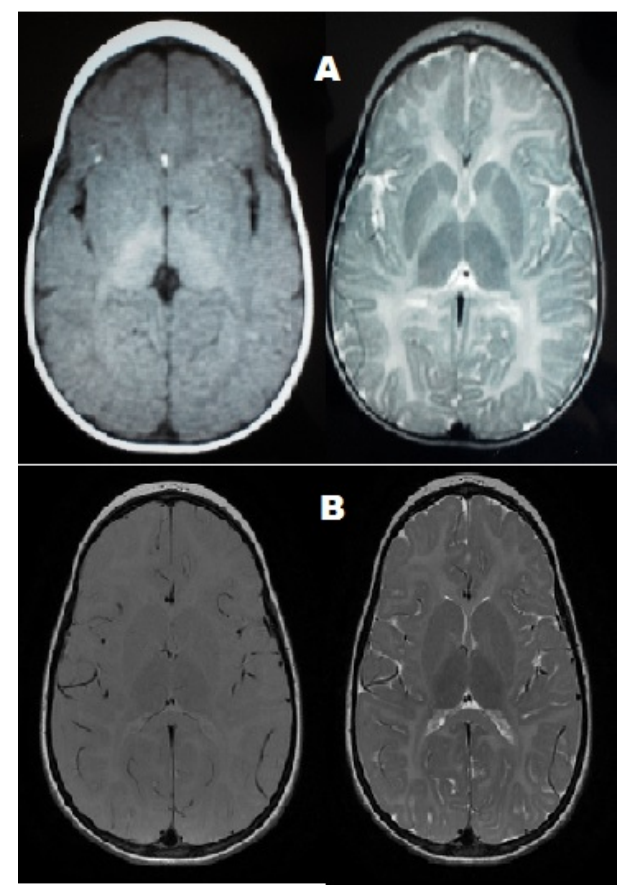

Figure 1: Patient's brain magnetic resonance imaging (MRI) showing severe demyelination at 9 months (Figure 1A) and 4 years old (Figure 1B).

\section{Discussion}

PMD is a neurodegenerative disease resulting in hypomyelinating or dysmyelinating patterns of the CNS white matter (leukodystrophy). It was first described in 1885 and later on in 1910 by German physicians Pelizaeus and Merzbacher who illustrated the clinical features of this disorder shared by 14 affected members of a family, including two females. Apart from these, it was noticed that PMD is inherited as Xlinked recessive disease. Males express the trait by showing early onset nystagmus and roving eye movements along with head nodding during infancy. Developmental milestones are delayed; choreoathetosis, ataxia and spasticity ultimately develop. Seizures occur only rarely in affected children 1. Death occurs in the second or third decade. MRI findings cannot distinguish PMD from other white matter disorders 2, while imaging features of hypomyelination may be apparent after one or two years of age, when the myelination process has been completed. In addition, auditory, visual and somatosensory evoked potentials are usually altered 1 . Medical therapy is limited to supportive care, such as physical therapy, orthotics, and antispasticity agents (i.e., baclofen).
Nonetheless, umbilical cord blood transplantation has been attempted with encouraging, though short-term, outcomes 3.

Definite confirmation of the disease is obtained usually through the demonstration of a PLP1 mutation although genomic alterations of novel genes [4] have also been found to be responsible.
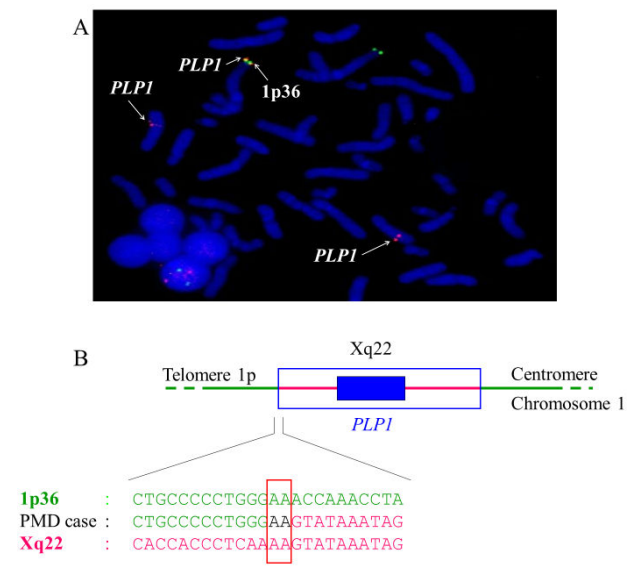

Figure 2: Insertion of an Xq22 segment including PLP1 in 1p36 chromosome. (2A): FISH analysis revealed that the additional PLP1 copy is inserted into $1 \mathrm{p} 36$ autosome (2B): Sequencing of a breakpoint of the insertion of PLP1-containing segment in 1p36 chromosome with a genome walking strategy revealed a two-base pair micro homology.

Cellular pathogenesis pertains to mutations of the proteolipid protein 1 gene $(P L P 1)$ in the $\mathrm{Xq} 22$ chromosome, resulting in truncation [5] of its protein products PLP and DM20, necessary for the CNS myelination process. Consequently, misfolded proteins trigger an endoplasmic reticulum (ER) stress in the oligodendrocytes that activates the unfolded protein response (UPR) [5,6]. The UPR is initially protective, but in situations of prolonged unresolved stress the UPR can lead to the apoptotic death of the myelinating glia.

Despite the distinctive and progressive features, a large clinical spectrum exists with some correlation between the type of mutation and the phenotype [7]. Two-thirds of the cases carry PLP1 duplications resulting in the classical-intermediate PMD form. Missense mutations give rise to more severe forms of the disease (connatal), whereas deletions and null mutations to mild PMD and spastic paraplegia type 2 (SPG2), a milder PMD allelic disorder. Other rare PLP1 abnormalities have been also identified, including X-chromosome translocations, triplications and partial duplications.

In our case, the patient was female and still expressed the disease even though females are mostly simple carriers. It has been clearly described that heterozygous females may be affected by an X-linked recessive trait usually through skewed $\mathrm{X}$-inactivation, and thus accumulation of the mutation-carrying $X$ chromosome [8]. Even though $\mathrm{X}$ chromosome inactivation studies failed to prove the mechanism above, high clinical suspicion due to classic manifestations of PMD disease did lead to further genetic investigation. Indeed, aCGH and FISH analysis verified the insertion of a PLP1 extra copy in 
Citation: Krepis P, Nikolaidou M, Maritsi D, Masliah-Planchon J, Boespflug-Tanguy O, et al. (2016) Severe Hypotonia, Nystagmus and Hypomyelination in A 9-Month Female Infant: Diagnosing Pelizaeus - Merzbacher Disease Outside the Usual Inheritance Patterns. J Neurol Disord 4: 301. doi:10.4172/2329-6895.1000301

Page 3 of 3

the autosomal loci 1p36 [9]. Eventually, a total gain of 3 copies of the referred gene was proved irrespective of the X-inactivation pattern. Hence, a functional PLP1 segment triplication can explain patient's phenotype and endorse the PMD diagnosis in a female. After a review of the literature, we located one other case of PMD affected female with such an unbalanced chromosomal translocation of PLP1 into 1 p36 [10]. PLP1 triplications have already been described [7] and are often associated with a more severe phenotype. Nevertheless, in our patient the triplicated segment did not include the entire PLP1 gene, but neighboring regions embedded in the PLP1 duplication [9]. This result suggests a milder phenotype that is consistent with our case.

\section{Conclusion}

Although PMD is an X-linked disease, females may be affected in various genetic mechanisms. Thus high clinical suspicion has to be maintained when nystagmus, hypotonia and developmental delay are present in infants, regardless of sex of the patient. Physicians should be aware of that possibility when they come across the typical PMD manifestations. A thorough genetic survey is crucial, not only for the precise PMD diagnosis, but also because it can illuminate the underlying genetic mechanism that may sometimes be inconsistent with the conventional genetic patterns.

\section{References}

1. Espinosa E, Mera-Solarte PR, Cote-Orozco JE (2016) Clinical features and diagnosis of Pelizaeus-Merzbacher disease: five case reports. Rev Neurol 62: 411-414.
2. Miyatake C, Koizumi S, Narazaki H, Asano T, Osaka H, et al. (2015) Clinical pictures in Pelizaeus-Merzbacher disease: a report of a case. J Nippon Med Sch 82: 74-75.

3. Page K, Wood S, Galvin L, Provenzale J, Escolar M (2014) Umbilical cord blood transplantation to treat Pelizaeus-Merzbacher disease in 2 young boys. Pediatrics 134: e1451-e14517.

4. Ji T, Li D, Wu Y, Xiao J, Ji H, et al. (2016) Identification of GJC2 gene mutations in Chinese patients with Pelizaeus-Merzbacher-like disease. Minerva Pediatr.

5. Dhaunchak AS, Colman DR, Nave KA (2011) Misalignment of PLP/ DM20 transmembrane domains determines protein misfolding in Pelizaeus-Merzbacher disease. J Neurosci 31: 14961-14971.

6. Clayton BL, Popko B (2016) Endoplasmic reticulum stress and the unfolded protein response in disorders of myelinating glia. Brain Res P II: 30180-30189.

7. Yamamoto T, Shimojima $\mathrm{K}$ (2013) Pelizaeus-Merzbacher disease as a chromosomal disorder. Congenit Anom (Kyoto) 53: 3-8.

8. Ørstavik KH (2006) Skewed X-inactivation in healthy individuals and in different diseases. Acta Paediatr Suppl 95: 24-29.

9. Masliah-Planchon J, Dupont C, Vartzelis G, Trimouille A, Eymard-Pierre E, et al. (2015) Insertion of an extra copy of Xq22.2 into $1 \mathrm{p} 36$ results in functional duplication of the PLP1 gene in a girl with classical PelizaeusMerzbacher disease. BMC Med Genet 16: 77.

10. Yiu EM, Farrell SA, Soman T (2009) Classic Pelizaeus-Merzbacher disease in a girl with an unbalanced chromosomal translocation and functional duplication of PLP1. Mov Disord 24: 2171-2172. 